\title{
I DIRITTI FONDAMENTALI SOCIALI A PRESTAZIONI E I VINCOLI DI BILANCIO DELLA COSTITUZIONE BRASILIANA
}

\section{OS DIREITOS FUNDAMENTAIS SOCIAIS A PRESTAÇÕES E OS VÍNCULOS ORÇAMENTÁRIOS NA CONSTITUIÇÃO BRASILEIRA}

\author{
Alexandra Cristina Giacomet Pezzi \\ Pubblico Avvocato del Comune di Porto Alegre/RS/Brasile. Dottore presso 1'Università Degli Studi Roma \\ Tre.
}

RIASSUNTO: $\mathrm{Fu}$, per molto tempo, messa in discussione l'essenzialità dei diritti sociali. La discussione è superata, sia in Brasile, sin in Italia. Nell'ordinamento giuridico brasiliano, i diritti sociali sono formalmente e materialmente costituzionali; dotati di applicabilità immediata e, inoltre, - nel caso della salute e dell'istruzione -, protetti da vincoli di bilancio.

Pertanto, ai diritti sociali non possono essere negati esigibilità e giustiziabilità, nonostante si riconosca che la sua realizzazione è molto più complessa che quella delle altre categorie."

Parole-chiave: Stato Sociale e Democratico di Diritto. Diritti sociali. Bilancio pubblico. Vincoli di bilancio.

\begin{abstract}
Foi, por longo tempo, questionada a fundamentalidade dos direitos sociais. A discussão está superada, seja no Brasil, seja na Itália. No ordenamento jurídico brasileiro, são os direitos sociais formal e materialmente constitucionais; dotados de aplicabilidade imediata e, ainda - no caso da saúde e da educação -, protegidos por vínculos orçamentários. Portanto, aos direitos sociais não podem ser recusadas exigibilidade e justiciabilidade, muito embora se reconheça que a sua concretização é bem mais complexa que a das demais categorias.

Palavras-chave: Estado Social e Democrático de Direito. Direitos sociais. Orçamento público. Vínculos
\end{abstract} orçamentários.

\section{Introduzione e evoluzione storica a partire dello Stato liberale}

Tutto lo svolgimento del presente studio sarà intriso dell'idea secondo la quale lo Stato ha un carattere eminentemente strumentale.

Secondo questa linea di pensiero, si sostiene che esso non costituisce un fine a sé stesso, ma che la sua esistenza ha come scopo quello di permettere che l'uomo raggiunga il suo completo destino.

Si ritiene che la massimizzazione del benessere - per tutti e non solo per una parte della popolazione - può essere raggiunta attraverso l'effettiva partecipazione dello Stato, sotto forma di prestazione di servizi pubblici di qualità.

L'avvento dell'era costituzionale, iniziata con la promulgazione della Costituzione statunitense del 1787, impose una disciplina all'azione statale, con la demarcazione dei poteri e la dichiarazione dei diritti fondamentali. 
L'articolo 16 della Dichiarazione della Rivoluzione Francese del 1789 aggiunse che "Qualsiasi società in cui non venga assicurata la garanzia dei diritti e neppure stabilita la separazione dei poteri, non possiede una Costituzione."

Il riconoscere nuovi diritti fondamentali, che concedono agli individui dei servizi come l'istruzione, la sanità, il lavoro e l'assistenza sociale, operò la transizione delle libertà formali astratte verso le libertà materiali concrete.

Lo Stato sociale di diritto si manifestò specialmente a partire dalla Costituzione messicana del 1917 e dalla Costituzione tedesca del 1919, nelle quali, per la prima volta, constavano chiaramente i diritti sociali. Mentre la Costituzione degli Stati Uniti Messicani assicurava diritti agrari, al lavoro e all'istruzione, quella tedesca andò oltre, garantendo casa, assistenza alle madri e aiuto alle famiglie numerose. ${ }^{1}$

Dopo la Seconda Guerra Mondiale, si intensificò la preoccupazione delle nazioni con il problema dei diritti sociali. Nel 1948, la Dichiarazione Universale dei Diritti dell'Uomo, redatta dall'Organizzazione delle Nazioni Unite, consacrò per la prima volta a livello internazionale, i diritti economici, sociali e culturali, successivamente specificati nel Patto Internazionale dei Diritti Economici, Sociali e Culturali del 1966.

Tuttavia, anche all'interno dello Stato sociale di diritto il problema della concretizzazione dell'uguaglianza non ottenne una piena soluzione, nonostante abbia superato la concezione puramente formale che caratterizzò lo Stato liberale.

Dallo Stato sociale si passò allo Stato democratico di diritto. Anche in esso si conferisce grande importanza alla legge, ma qui essa è vista come uno strumento di intervento nei confronti della realtà sociale, visto che l'idea di democrazia è intimamente legata alla questione delle condizioni materiali dell'esistenza umana.

Tra la democrazia e l'uguaglianza esiste un nesso profondo. L'uguaglianza cercata attraverso il sistema costituzionale è l'uguaglianza materiale, ossia, non solo dinanzi alla legge, ma attraverso la legge. ${ }^{2}$

\footnotetext{
${ }^{1}$ Andrea Rovagnati spiega che le Costituzioni precedenti a quella di Weimar non contemplavano i diritti sociali né esplicitamente né implicitamente perché "i principi in esame non solo non erano stati ancora proclamati in alcun documento giuridico ma nemmeno idealmente concepiti". Pensatori come Bodin, Hobbes, Locke, Rousseau, Montesquieu, Kant e tanti altri è che svilupparono l'idea di auto-determinazione dell'individuo e di che il compito principale del potere pubblico è riconoscere ai singoli individui una sfera relativamente ampia di libertà giuridica in quasi tutti i settori in cui si svolge la vita dell'uomo, soprattutto in quella economica, la quale trovò un vasto consenso in ambito culturale, sociale e politico, e favorì una grandiosa opera di rinnovamento dei sistemi giuridici, con la approvazione di testi normativi, come Costituzioni e Carte dei diritti. (ROVAGNATI, Andrea. Sulla natura dei diritti sociali. Torino: Giappichelli, 2009, p. 2-3).

${ }^{2}$ Secondo Vieira de Andrade, non si tratta di un'uguaglianza formale, astratta, livellatrice e unificatrice, ma dell'uguaglianza materiale, che esige la considerazione della realtà nella quale le persone si muovono e, di conseguenza, la diversità di trattamento e di statuto di quelli che, per le proprie qualità specifiche o per la loro
} 
Allo stesso modo in cui l'uguaglianza nei diritti crea il senso di uguaglianza basata nel rispetto dell'altro come uguale, la disuguaglianza nei diritti fa sorgere l'immagine dell'altro come disuguale, cioè, inferiore sul piano antropologico, proprio perché inferiore sul piano giuridico. ${ }^{3}$

Lo Stato democratico progredì rispetto alle proposte che lo precedettero, considerando che l'uguaglianza deve essere intesa anche in senso sostanziale, in quanto ogni società contiene al suo interno disuguaglianze di fatto, che debbono essere rimosse nella misura in cui impediscono il pieno sviluppo della persona umana e l'effettiva partecipazione di tutti i lavoratori all'organizzazione politica, economica e sociale del paese. Secondo questa prospettiva, il principio di uguaglianza rappresenta il punto di contatto tra lo Stato democratico e lo Stato sociale: l'uno realizza e dà sostanza all'uguaglianza giuridica, l'altro si propone di offrire a tutti i cittadini quelle parità di opportunità e di condizioni che la società, in ragione della sua struttura, non è in grado di predisporre autonomamente. Il principio di uguaglianza sostanziale opera a favore dei cosiddetti "soggetti deboli", i quali vedono ostacolata per ragioni economiche e sociali la possibilità di un esercizio effettivo e paritario dei diritti costituzionali: la donna rispetto all'uomo, il lavoratore rispetto al datore di lavoro, gli emarginati sociali rispetto agli strati più inseriti. ${ }^{4}$

Gli interventi statali pretendono, in ultima analisi, di garantire a tutti i cittadini le condizioni minime per una vita degna. Il minimo esistenziale è imprescindibile, qualsiasi sia l'organizzazione sociale che sarà implementata. ${ }^{5}$

\subsection{La formazione della Repubblica Federativa del Brasile: il percorso costituzionale e il consolidamento dei diritti sociali}

L'analisi della storia del Brasile rivela che la sua esperienza di libertà non è lunga.

\footnotetext{
situazione nel processo sociale, hanno bisogno di una protezione (differente) necessaria e adeguata alla loro (pari) dignità di persone. Comunque, si penserà a tutti, nell'universalità, e non in alcuni, nella particularità. L'idea è che l'universalizzazione dei diritti sociali è l'ultima tappa dello Stato Sociale. (ANDRADE, José Carlos Vieira de. Os direitos fundamentais na Constituição portuguesa de 1976. 3. ed. Coimbra: Almedina, 2004, p. 139-140).

${ }^{3}$ FERRAJOLI, Luigi. Derechos y garantias. La ley del más débil. Madri: Ed. Trotta, 1999, p. 58.

${ }^{4}$ ROLLA, Giancarlo. La tutela costituzionale dei diritti. V. III. 3. ed. Milano: Giuffrè, 2007, p. 50.

5 Per John Rawls, senza il minimo sociale, inteso come una situazione di pari opportunità, non esiste una fruizione effettiva dei diritti di libertà, come pure non consente qualsiasi giustizia distributiva. Il minimo esistenziale o sociale costituirebbe, pertanto, secondo l'autore, la vera condizione di libertà. (RAWLS, John. Liberalismo Político. Tradução de Sergio René Madero Báez. México: Fondo de Cultura Econômica, 1992, p. 218). Abbiamo avuto l'opportunità di trattare il minimo esistenziale nel nostro Dignidade da pessoa humana, mínimo existencial e limites à tributação no Estado Democrático de Direito, pubblicato dalla casa editrice Juruá.
} 
Subito dopo la dominazione coloniale, che si è protratta dal 1500, anno della "scoperta" della nazione da parte dei portoghesi ${ }^{6}$, fino al 1822, ebbe inizio l'Impero.

L'Assemblea Costituente formata da rappresentanti delle diverse provincie fu sciolta da Dom Pedro I che, il 25 marzo 1824, ordinò una costituzione intitolata "Costituzione Politica dell'Impero del Brasile”. Il percorso costituzionale brasiliano, come si può vedere, cominciò sotto il segno di un'imposizione.

Nonostante contenesse un lungo elenco di "garanzie dei diritti civili e politici dei cittadini brasiliani" 7 e consacrasse i princìpi della legalità e dell'uguaglianza ${ }^{8}$, la Carta del 1824, senza rompere con la tradizione lusitana, istituzionalizzò, con diversi strumenti, il modello oligarchico, che conservava con la struttura normativa della Costituzione un rapporto meramente formale.

Erano tollerati sia i privilegi della nobiltà sia il voto secondo il reddito ${ }^{9}$ e il regime della schiavitù. Buona parte della popolazione era composta da schiavi, considerati "cose" invece che "soggetti di diritto".

L'eredità dell'Impero è estremamente chiarificatrice: $83 \%$ di analfabeti, su una popolazione di poco più di 14 milioni di persone. È curioso che la Costituzione del 1824 garantiva, nel suo articolo 179, comma XXXII, "L'istruzione primaria e gratuita a tutti i Cittadini". ${ }^{10}$

Solamente il 13 maggio 1888 fu abolito il lavoro schiavo. ${ }^{11}$ Il Brasile, purtroppo, fu l'ultimo paese di tradizione cristiana e occidentale a liberarli, non facendolo per convinzione, ma per convenienza. Confermò appena la sua obsolescenza dinanzi ai nuovi rapporti capitalisti di produzione.

\footnotetext{
${ }^{6}$ Nell'idea di Heródoto Barbeiro, "Non ci sono dubbi che la scoperta del Brasile fu intenzionale. Questa tesi può essere dimostrata se consideriamo il dominio lusitano del Mar Oceano, che allontana l'ipotesi di un cambio della rotta per una bonaccia. Il Trattato de Tordesillas e la mancata accettazione della Bula Inter Coetera da parte dei portoghesi dimostrarono la piena conoscenza del Portogallo sulla regione." (BARBEIRO, Heródoto. Curso de história do Brasil. São Paulo: Harbra, 1946, p. 9).

${ }^{7}$ Come esempio, si fa riferimento all'articolo 178, caput: "L'inviolabilità dei Diritti Civili e Politici dei Cittadini Brasiliani, che hanno come base la libertà, la sicurezza individuale e la proprietà, è garantita dalla Costituzione dell'Impero, nella seguente maniera:[...]"

${ }^{8}$ Ciò è stato previsto dal comma XIII dell'articolo 179: "La Legge sarà uguale per tutti, che protegga, che castighi, o ricompenserà in modo proporzionale ai meriti di ciascuno."

${ }^{9}$ Il voto, oltre a essere maschile, era secondo il reddito: per votare, era necessario possedere un determinato reddito annuale. Anche per essere un candidato a deputato o senatore era richiesto un reddito alto.

${ }^{10}$ BARROSO, Luís Roberto. O direito constitucional e a efetividade de suas normas. 8. ed. Rio de Janeiro: Renovar, 2006, p. 9-12.

${ }^{11}$ Attraverso la cosiddetta "Lei Áurea", sanzionata dalla Reggente Principessa Isabel. Essa fu preceduta dalla "Lei do Ventre Livre", che considerava i figli nati da schiavi come liberi, e, nel 1885, la "Lei do Sexagenário", che concedeva la libertà agli schiavi com più di sessanta anni d'età.
} 
Venne così stimolata l'immigrazione europea, persino dai pubblici poteri, "interessati a sostituire l'oneroso lavoro degli schiavi con il più vantaggioso lavoro salariato (maggior produzione e miglior qualità)". ${ }^{2}$

Fu particolarmente intensa l'immigrazione italiana, che si protrasse dal 1870 fino all'inizio del ventesimo secolo. Si diresse specialmente alle piantagioni di caffè dello Stato di San Paolo, ma un gran numero di immigranti si sparse a Sud e Sud Est del Brasile. Si spiega: il Sud continuava poco popolato e nella mira delle nazioni vicine, per questo il governo stimolò l'insediamento degli immigranti.

Fatta questa digressione storica, è necessario analizzare il periodo successivo alla promulgazione della Costituzione del 24 febbraio 1891, ispirata al modello nordamericano. Essa operò una triplice trasformazione: 1. forma di governo: si passò da monarchia a repubblica; 2. sistema di governo: da parlamentare in presidenziale; 3. forma di Stato: da unitaria fu convertita in federale. ${ }^{13}$

Durante la $1^{\text {a }}$ Repubblica, che durò dal 1889 al 1930, si continuò, però, a ignorare completamente i diritti sociali. La Costituzione assicurò solo, nel capitolo denominato "Dichiarazione dei diritti", i diritti tipicamente liberali, come libertà, sicurezza individuale e proprietà.

Spettò alla Costituzione del 1934, influenzata dalla Costituzione di Weimar del 1919, di introdurre importanti innovazioni. Dedicò, pertanto, un titolo specifico all'Ordine Economico e Sociale, dando inizio all'era dell'intervento statale. Nel suo articolo 115, si legge:

Art. 115 - L'ordine economico deve essere organizzato secondo i principi della Giustizia e delle necessità della vita nazionale, in modo che permetta a tutti un'esistenza degna. All'interno di questi limiti, è garantita la libertà economica.

Creò, inoltre, la Giustizia del Lavoro e lo stipendio minimo. Nel suo articolo 113, comma 34, garantiva a tutti il diritto di provvedere al proprio sostentamento e a quello della propria famiglia mediante un lavoro onesto, dovendo il Potere pubblico proteggere, ai sensi di legge, chi fosse in situazione di indigenza. Si rivela qui un'antica preoccupazione del legislatore nel garantire un minimo di sussistenza ai bisognosi.

\footnotetext{
${ }^{12}$ Il lavoro schiavo, molto vantaggioso nella fase iniziale di implementazione del ciclo della canna da zucchero e della produzione del caffè, diventò un problema, specialmente a causa della crescente pressione inglese contro la tratta dei negri africani verso il Brasile. L'interesse britannico, è bene dire, era soprattutto finanziario, poichè il passaggio al capitalismo liberale richiedeva una manodopera più adeguata alla nuova politica economica, cioè, la manodopera stipendiata. (BARBEIRO, Heródoto. Curso de história do Brasil. São Paulo: Harbra, 1946, p. 172 a 180).

${ }^{13}$ BARROSO, Luís Roberto. O direito constitucional e a efetividade de suas normas. 8. ed. Rio de Janeiro: Renovar, 2006, p. 13.
} 
Trattò del diritto alla sindacalizzazione e fissò una lista di diritti dei lavoratori. Attribuì all'Unione e agli Stati la competenza per legiferare in merito alla sanità. Definì il diritto all'istruzione come un diritto di tutti, garantendo la gratuità della scuola primaria e la tendenza alla gratuità dopo questo livello. Infine, ampliò le forme pensionistiche nell'ambito della pubblica amministrazione.

A partire dal 1937, i diritti sociali dei lavoratori e alcuni diritti alla previdenza furono riconosciuti e tutelati, malgrado il carattere paternalista da parte del governo e la soggezione dei sindacati al potere pubblico.

La sanità, a sua volta, doveva essere regolamentata esclusivamente da norme dell'Unione, ai sensi dell'articolo 16, comma XXVII.

Presentava un esteso catalogo di diritti del lavoro che esistono tutt'oggi. Malgrado la previsione di una serie di diritti sociali, è bene ricordare che il contesto era la dittatura - il cosiddetto Nuovo Stato -, instaurato da Getúlio Vargas subito dopo il colpo di stato del 1937, con l'imposizione di una Costituzione che, ispirata al fascismo, istituzionalizzò uno Stato totalitario, con la censura della stampa, il controllo delle masse popolari attraverso i sindacati vincolati al Ministero del Lavoro e un marcante interventismo statale. ${ }^{14}$

Con la fine della Seconda Guerra Mondiale, diversi regimi dittatoriali si conclusero; gli Stati indipendenti elaborarono i propri statuti fondamentali. Tra il 1946 e il 1949, ventun paesi stilarono nuove Costituzioni, senza contare l'America Latina, dove otto Nazioni inaugurarono nuove Leggi fondamentali o modificarono profondamente quelle esistenti. ${ }^{15} \mathrm{Fu}$ il caso del Brasile che, nel 1946, fece promulgare una nuova Costituzione, dando inizio al processo di "ridemocratizzazione".

La Costituzione del 1946 garantiva ai brasiliani e agli stranieri residenti nel paese l'inviolabilità dei diritti concernenti la vita, la libertà, la sicurezza individuale e la proprietà e prevedeva una serie di diritti del lavoro.

L'articolo 166 trattava dell'istruzione, proclamata come un diritto di tutti, da fornirsi nelle case e nella scuola. La difesa e lo sviluppo del diritto alla sanità e il sistema della previdenza furono messi a carico dell'Unione, nell' articolo 5, comma 15, lettera “b”.

Una preziosa regola fu inserita all'articolo 169: per la prima volta, la Costituzione stabilì una percentuale minima di risorse per la realizzazione di un diritto sociale, nell'esigere che l'Unione applicasse annualmente non meno che il dieci per cento e gli Stati, il Distretto

\footnotetext{
${ }^{14}$ BARBEIRO, Heródoto. Curso de história do Brasil. São Paulo: Harbra, 1946, p. 246-247.

${ }^{15}$ BARROSO, Luís Roberto. O direito constitucional e a efetividade de suas normas. 8. ed. Rio de Janeiro: Renovar, 2006, p. 25.
} 
Federale e i Comuni, non meno che il venti per cento delle entrate derivanti dalle imposte, per la manutenzione e lo sviluppo dell'istruzione.

Nel 1964, però, il paese subì un colpo di stato militare ${ }^{16}$, i cui effetti si protrassero fino al 1985. Pertanto, solo dopo circa vent'anni di dittatura, il Brasile rientrò nel gruppo dei paesi democratici, con l'insediamento di una Assemblea Nazionale Costituente per elaborare la nuova Costituzione della Repubblica.

Non sorprende che presenti ancora una fisionomia immatura, frutto delle successive rotture istituzionali. La realtà socio-politica trova nella storia una giustificazione.

Non ci sono dubbi che la Costituzione federale del 1988 introdusse grandi innovazioni, soprattutto nel campo dei diritti fondamentali.

A dispetto di una norma esplicita nel nuovo testo costituzionale, gli autori sono unanimi nell'affermare che la Repubblica Federativa del Brasile adottò il modello di Stato sociale. Tanto è vero che, nel suo articolo 3, enumera come obiettivi fondamentali la costruzione di una società libera, giusta e solidale; la garanzia dello sviluppo nazionale; l'eliminazione della povertà e dell'emarginazione, la riduzione delle disparità sociali e regionali; la diffusione del benessere, senza preconcetti o discriminazioni. Tutto il sistema di pianificazione stabilito dalla Costituzione per lo sviluppo nazionale deve essere diretto al raggiungimento di questi obiettivi ${ }^{17}$, da concretizzarsi attraverso politiche pubbliche di sviluppo e di inserimento sociale.

Purtroppo, la Costituzione del 1988 ha già subito oltre ottanta emendamenti, alcuni lesivi il sistema di valori tracciato dal legislatore costituente. È realmente più difficile, per una società segnata dall'autoritarismo, redigere una Costituzione a carattere definitivo. Non si può, tuttavia, non evidenziare quanto delle riforme periodiche sono fonte di incertezza, mentre uno degli assi principali su cui deve gravitare lo Stato di diritto è la sicurezza dei rapporti

\footnotetext{
${ }^{16}$ L'atto istituzionale del 9 aprile 1964, emesso dai comandanti in capo delle Forze Armate, determinò le seguenti misure: elezione indiretta del presidente della repubblica; sospensione delle garanzie dei vitalizi e della stabilità; possibilità di licenziamento; esenzione o pensione di dipendenti pubblici federali, statali e municipali; possibilità di annullamento dei diritti politici e dei mandati legislativi, tra le altre cose a carattere eminentemente discrezionale. Durante il regime militare post 1964 , le restrizioni al voto, le persecuzioni nei confronti dell'opposizione, l'annullamento dei mandati e l'uso della repressione fisica furono elementi costanti della vita politica nazionale.

${ }^{17}$ Secondo Konrad Hesse, "Nonostante la Costituzione non possa, da sola, realizzare nulla, può imporre compiti. La Costituzione si trasforma in una forza attiva se queste azioni sono effettivamente realizzate, se esiste la disponibilità a orientare la propria condotta secondo l'ordine in essa stabilita, se, a dispetto di tutte le domande e riserve provenienti dai giudizi di convenienza, si può identificare la volontà di concretizzare quest'ordine. Concludendo, si può affermare che la Costituzione si convertirà in forza attiva se si faranno presenti, nella coscienza generale - in particolare, nella coscienza dei principali responsabili dell'ordine costituzionale -, non solo la volontà del potere (Wille zur Macht), ma anche la volontà della Costituzione (Wille zur Verfassung). (HESSE, Konrad. A Força Normativa da Constituição. Tradução de Gilmar Ferreira Mendes. Porto Alegre: Fabris, 1991, p. 19).
} 
giuridici. Quest'orbita disarmonica provoca l'allontanamento di un'altra coordinata fondamentale del sistema: la giustizia. Naturalmente un ordine giuridico incapace di soddisfare questi due valori fondamentali si riduce a un mero formalismo retorico.

Sotto l'aspetto sociologico, la discontinuità istituzionale frustra la cristallizzazione di un sentimento costituzionale, "risultato ultimo dell'entrata della Legge fondamentale nella vita quotidiana dei cittadini, creando una coscienza comunitaria di rispetto e preservazione, come un simbolo superiore, di valore affettivo e pragmatico". ${ }^{18}$

Non senza ragione mancano ai brasiliani un sentimento democratico e una coscienza nazionale. Il binomio Stato forte - società civile debole lasciò molti strascichi, come rivela l'attuale contesto socio-politico.

Il primo presidente eletto democraticamente, dopo anni di dittatura, subì un impeachment. Numerose altre denuncie di corruzione vengono fatte quasi giornalmente, colpendo gran parte delle sfere di governo e degli altri Poteri dello Stato.

L'abituale dichiarazione di assenza di risorse destinate ai settori della sanità e dell'istruzione è smentita dalle notizie di ripetuti record di riscossione a livello federale, rivelando l'inefficienza e le incoerenze del sistema. Si coniugano, in maniera perversa, miseria, corruzione e spreco. Le classi più bisognose della popolazione continuano a non avere accesso al mercato formale del lavoro, anche per mancanza di qualifica.

Questo scenario causa l'aggravamento delle distorsioni nella distribuzione del reddito nel paese a livelli veramente inaccettabili.

Inserito nell' economia mondiale come uno dei dieci grandi produttori di ricchezze, il Brasile continua a convivere con indici insoddisfacenti in aree essenziali come l'istruzione, l'abitazione e la sanità.

Insomma, la storia del Brasile, segnata da lunghi periodi di autoritarismo, ma anche il suo attuale contesto politico di miseria e di disparità, sono fattori che spiegano l'apatia politica, incompatibile con la formazione della cittadinanza.

La nozione di cittadinanza è oggi associata a una dimensione spiccatamente attiva di partecipazione. Non è possibile pretendere la realizzazione dei diritti fondamentali senza una nuova concezione di cittadinanza che non implichi solo il "votare ed essere votato".

\footnotetext{
18 BARROSO, Luís Roberto. O direito constitucional e a efetividade de suas normas. 8. ed. Rio de Janeiro: Renovar, 2006, p. 47-48. Secondo lo stesso autore, "Costituzioni, com'è volgarmente noto, non ci sono mancate. Al contrario, in questa materia avremo peccato più per eccesso che per difetto. [...] Così, in una successione di ostacoli, furono redatte, in poco più di 180 anni di Indipendenza e 110 di Repubblica, otto Costituzioni, in un malinconico segno di instabilità e mancanza di continuità delle nostre istituzioni politiche". (BARROSO, 2006, p. 7-8).
} 
L'esercizio della cittadinanza richiede un rinnovamento del soggetto, quando questi inizia a percepirsi come responsabile del processo del cambiamento sociale. ${ }^{19}$

\section{L'essenzialità e il significato dei diritti sociali}

L'essenzialità dei diritti sociali fu per molto tempo contestata sia in Italia che in Brasile e anche in altri ordinamenti giuridici.

Gli autori che si oppongono al loro riconoscimento come autentici diritti fondamentali abitualmente sostengono che la maggior parte dei diritti sociali è enunciata per mezzo di norme programmatiche e che per questo sono sprovvisti di vincolo giuridico, rappresentando un mero appello alle istanze politiche. ${ }^{20} \mathrm{Ci}$ sono anche quelli che riducono il loro carico di essenzialità appena al cosiddetto minimo esistenziale. ${ }^{21}$

È possibile affermare che tale discussione è superata. Oggi si sa che i diritti sociali rientrano a pieno titolo nel novero dei diritti fondamentali e che costituiscono anche "elementi qualificanti la forma di Stato (Stato sociale democratico di diritto), per cui sono anche principi orientatori dell'attività dello Stato". ${ }^{22}$

Da ciò la duplice natura dei diritti sociali, i quali rappresentano, nel medesimo tempo, diritti veri e propri e disposizioni costituzionali di principio.

Nella Costituzione italiana, i diritti sociali possono essere ricondotti sia all'articolo 2, che riconosce i diritti inviolabili della persona, sia all'articolo 1, il quale qualifica il carattere repubblicano dello Stato, che all'articolo $3 .^{23}$

\footnotetext{
${ }^{19}$ COSTA, Marli M. da. O discurso no espaço local para a concretização da cidadania de crianças e jovens frente à exclusão social. In: REIS, Jorge Renato dos; LEAL, Rogério Gesta (orgs). Direitos Sociais e políticas Públicas. Desafios Contemporâneos. T. 7, Santa Cruz do Sul: EDUNISC, 2007, p. 1883.

${ }^{20}$ Come il riconoscimento legislativo dei diritti sociali è avvennuto all'interno di ordinamenti di modello liberale, è comprensibile la tendenza, comune tra i giuspubblicisti dell'epoca, a considerare tali diritti meramente come espressione di scelte di legislatori ispirati a principi politici di giustizia sociale, e a giudicarli privi di una loro specificità istituzionale. (ROVAGNATI, Andrea. Sulla natura dei diritti sociali. Giappichelli: Torino, 2009, p. 16). Secondo Baldassarre, in Italia, nel corso degli anni'50, la Corte di Cassazione sposò la tesi relativa alla programmaticità di tutte le norma costituzionali, ma questa fu abbattuta dalla Corte costituzionale (BALDASSARRE, Antonio. La normatività della Costituzione e i suoi "nemici". Rivista di Diritto Costituzionale,Torino, Giappichelli, 1996, p. 10).

${ }^{21}$ È il caso di Ricardo Lobo Torres, secondo il quale ai diritti sociali manca l'essenzialità, salvo quando rappresentano il minimo necessario all'esercizio della libertà. L'autore riduce, pertanto, la fondamentalità dei diritti sociali al cosiddetto minimo esistenziale. (TORRES, Ricardo Lobo. Tratado de Direito Constitucional Financeiro e Tributário. Os Direitos Humanos e a Tributação: Imunidades e Isonomia. V. III. 3. ed. Rio de Janeiro: Renovar, 2005, p. 169 e seguenti).

${ }^{22}$ ROLLA, Giancarlo. La tutela costituzionale dei diritti. V. III. 3. ed. Milano: Giuffrè, 2007, p. 26.

${ }^{23}$ ROLLA, Giancarlo. La tutela costituzionale dei diritti. V. III. 3. ed. Milano: Giuffrè, 2007, p. 26. Gli articoli 1,2 e 3 della Costituzione italiana enunciano che: " 1 L'Italia è una Repubblica democratica, fondata sul lavoro. La sovranità appartiene al popolo, che la esercita nelle forme e nei limiti della Costituzione"; "2 La Repubblica riconosce e garantisce i diritti inviolabili dell'uomo, sia come singolo sia nelle formazioni sociali ove si svolge la sua personalità, e richiede l'adempimento dei doveri inderogabili di solidarietà politica, economica e sociale"; "3
} 
I diritti sociali, al pari delle libertà fondamentali, sono sottratti alla disponibilità non solo del legislatore ordinario ma anche a quella del legislatore costituzionale.

Anche per quanto riguarda la Costituzione brasiliana non vi sono dubbi che l'opzione del legislatore fu quella di considerare i diritti sociali come autentici diritti fondamentali, sia nell' aspetto formale o materiale, tanto è vero che la maggior parte di questi diritti è prevista nell'articolo 6 della Costituzione, che integra il Titolo II, riservato ai diritti e alle garanzie fondamentali.

L'esame degli articoli 1 e 3 del testo costituzionale, relativi ai fondamenti e agli obiettivi della Repubblica Federativa del Brasile, rivela che i diritti sociali sono anche materialmente fondamentali.

Pertanto, hanno una protezione rafforzata contro un'eventuale riforma. Nell'articolo 60, paragrafo 4 della Costituzione federale, constano le cosiddette "clausole di pietra", con il seguente testo:

Art. 60. La Costituzione potrà essere emendata mediante proposta:

I - di, come minimo, un terzo dei membri della Camera dei Deputati o del Senato Federale;

II - del Presidente della Repubblica;

III - di oltre la metà delle Assemblee Legislative delle unità della Federazione, manifestandosi, ognuna, per maggioranza relativa dei loro membri.

$\S 4^{\circ}$ - Non sarà oggetto di delibera la proposta di emendamento tendente ad abolire:

I - la forma federativa dello Stato;

II - il voto diretto, segreto, universale e periodico;

III - la separazione dei poteri;

IV - i diritti e garanzie individuali.

Di conseguenza, il legislatore non possiede una libertà illimitata di conformazione nel procedere alla riforma della Costituzione, sia in ragione dell'identità, sia in ragione della posizione gerarchica della Costituzione nell'ordine giuridico. ${ }^{24}$

I diritti fondamentali, secondo il citato articolo, costituiscono limiti materiali espliciti. Tuttavia,

\begin{abstract}
continua controversa l'inclusione di tutti i diritti fondamentali nell'elenco dei limiti materiali (espliciti o impliciti), visto che la Costituzione, nel suo articolo $60, \S 4^{\circ}$, comma IV, si riferisce ai 'diritti e garanzie individuali' e ciò ha creato, da parte di non pochi studiosi, un rifiuto (o, perlomeno, una certa riserva) rispetto alle condizioni di limiti materiali alla riforma costituzionale da parte dei diritti fondamentali sociali. ${ }^{25}$
\end{abstract}

Tutti i cittadini hanno pari dignità sociale e sono eguali davanti alla legge, senza distinzione di sesso, di razza, di lingua, di religione, di opinioni politiche, di condizioni personali e sociali. È compito della Repubblica rimuovere gli ostacoli di ordine economico e sociale, che, limitando di fatto la libertà e l'eguaglianza dei cittadini, impediscono il pieno sviluppo della persona umana e l'effettiva partecipazione di tutti i lavoratori all'organizzazione politica, economica e sociale del Paese."

${ }^{24}$ SARLET, Ingo. Os direitos fundamentais sociais como "cláusulas pétreas". Revista de Interesse Público, Porto Alegre, n. 17, 2003, p. 59.

${ }^{25}$ SARLET, Ingo. Os direitos fundamentais sociais como "cláusulas pétreas". Revista de Interesse Público, Porto Alegre, n. 17, 2003, p. 65-66. 
Pensiamo che, se le dette "clausole di pietra" proteggono proprio "l'insieme di beni costituzionali essenziali alla preservazione dell'identità della Costituzione”, per forza comprendono anche i diritti fondamentali sociali. La protezione dev'essere massima, nel senso che deve comprendere tutti i diritti fondamentali, anche quando al di fuori del Titolo II. ${ }^{26}$ Il Supremo Tribunale Federale si sta posizionando per conferire un'interpretazione estensiva alla categorie delle "clausole di pietra", inserendo in esse i diritti fondamentali sociali. $^{27}$

Anche la giurisprudenza e la dottrina italiane contemporanee prevalenti riconoscono ai diritti sociali (quale che essi siano) il carattere di diritti inviolabili o fondamentali. ${ }^{28}$

L'oggetto tipico dei diritti sociali è il cosiddetto "minimo di esistenza materiale" (materielle Existenzminimum), come misura di minor grado di protezione nelle aree di educazione e sanità, per esempio. ${ }^{29}$

Ciò, tuttavia, non significa che uno Stato possa rassegnarsi a offrire sempre prestazioni minime ai propri abitanti. È obbligato a diffondere l'incremento delle condizioni di vita oltre questo livello. I diritti non possono essere screditati: devono essere presi sul serio.

\footnotetext{
${ }^{26}$ SARLET, Ingo. Os direitos fundamentais sociais como "cláusulas pétreas". Revista de Interesse Público, Porto Alegre, n. 17, 2003, p. 73.

${ }^{27}$ Nell'azione diretta di incostituzionalità $\mathrm{n}^{\circ}$ 939, il Ministro del Supremo Tribunale Federale Carlos Velloso fece riferimento ai diritti sociali come appartenenti alla categoria dei diritti e garanzie individuali, pertanto, immutabili, mentre il Ministro Marco Aurélio affermò che i diritti sociali si inseriscono tra i diritti individuali previsti nell'articolo 60, comma 4 della Costituzione federale.

${ }^{28}$ Secondo Andrea Giorgis, "la dottrina è pressoché unanime nel riconoscere ai diritti sociali (quale che essi siano) il carattere di diritti inviolabili e/o fondamentali". (GIORGIS, Andrea. Diritti sociali. In: CASSESE, Sabino (coord.). Dizionario di Diritto Pubblico. V. III. Milano: Giuffrè, 2006, p. 1910). Rispetto ala tutela della salute è importante menzionare che l'articolo 32 della Costituzione italiana, inizialmente considerato soprattutto nell'aspetto pubblicistico, in quanto espressivo di un interesse della collettività, è stato in tal modo progressivamente valorizzato nel profilo che induce a considerare la salute anche quale oggetto di un diritto inviolabile dell'uomo, da inserire tra quelli garantiti dall'art. 2 della carta fondamentale. (Corte Suprema di Cassazione, Consiglio Superiore della Magistratura, 4 marzo 2009, incontro di studi sul tema "Profili problematici della tutela del diritto alla salute"). Nell'affermazione di Luigi Ferrajoli, non solo il diritto alla salute, come "tutti i diritti fondamentali, incluso il diritto all'istruzione, sono sottratti non solo alle decisioni di maggioranza, ma anche al mercato: sono inviolabili e inalienabili, inalterabili e indisponibili. Stabilire normativamente un diritto fondamentale vuol dire perciò sottrarlo al mercato". (FERRAJOLI, Luigi. Principia iuris. Teoria del diritto e della democrazia. T. 2, Bari: Laterza, 2007, p. 416-417). Sull'importanza della rigidità costituzionale per il controllo della maggioranza scrive Federico Sorrentino: "La rigidità costituzionale è una garanzia che si contrappone idealmente all'idea democratica, per la quale la maggioranza di oggi non può stabilire limiti alle maggioranze di domani. L'idea sottostante alla rigidità costituzionale è invece che, per aversi un libero gioco democratico, per evitare che la maggioranza che ha in mano il potere prevarichi e opprima le minoranze, impedendo loro di diventare in futuro maggioranza, siano necessarie norme sottratte alla maggioranza contingente e garanzie per la rigidità costituzionale stessa." (SORRENTINO, Federico. Le garanzie costituzionali dei diritti. Lezioni raccolte da Roberta Calvano. Torino: Giappichelli Editore, 1998, p. 48).
}

${ }^{29}$ QUEIROZ, Cristina. Direitos fundamentais sociais. Coimbra: Coimbra Editora, 2006, p. 49-50. In questo passaggio l'autrice fa riferimento alla teoria della "posizione minima definitiva", fissata da Gertrude LübbeWolff, Grundrechte als Eingriffsabwehrrechte. Struktur und Reich-weite der Eingriffsdogmatik im Bereich staatlicher Leistung, Baden-Baden, 1988, p. 161. 
La garanzia di un minimo sociale è destinata a evitare la perdita della funzione del diritto fondamentale, in modo che esso non risulti privo di contenuto. ${ }^{30}$

\section{La giustiziabilità dei diritti sociali}

Il comma 1 dell'articolo 5 della Costituzione brasiliana enuncia che "Le norme che definiscono diritti e garanzie fondamentali hanno applicazione immediata."

Succede, tuttavia, che questa applicabilità immediata avviene nella misura dell'efficacia di ciascuna di queste norme. Malgrado tutte le norme costituzionali possegano efficacia, si distinguono per il grado. ${ }^{31}$

La questione rese celebre la tripartizione elaborata da José Afonso da Silva, in un'opera classica, secondo quanto segue:

1. Norme costituzionali di efficacia piena e applicabilità diretta, immediata e integrale;

2. Norme costituzionali di efficacia limitabile e applicabilità diretta, immediata, ma possibilmente non integrale;

3. Norme costituzionali di efficacia limitata, dichiarative di principi istitutivi od organizzativi e dichiarative di principi programmatici. ${ }^{32}$

Questa classificazione è adottata dalla giurisprudenza prevalente del Supremo Tribunale Federale. ${ }^{33}$

Il terzo gruppo, quello delle norme di efficacia ridotta, o si limitano a enunciare principi, come l'articolo 196 della Costituzione federale ("La salute è diritto di tutti e dovere

\footnotetext{
${ }^{30}$ Ancora secondo Cristina Queiroz, "Questo 'standard minimo incondizionale', che è fissato, in Germania, dalla giurisprudenza del Tribunale Costituzionale Federale, non dovrà, però, essere interpretato in modo restrittivo, prima di essere progressivamente fissato e diffuso sotto una prospettiva aperta e particolare." (QUEIROZ, Cristina. O princípio da não-reversibilidade dos direitos fundamentais sociais. Princípios dogmáticos e prática jurisprudencial. Coimbra: Coimbra Editora, 2006, p. 93).

${ }^{31}$ Maria Helena Diniz fornisce una spiegazione didattica sulla questione, affermando che "Esiste una scala nell'intangibilità e negli effetti dei precetti costituzionali. [...] Tutte hanno giuridicità, ma sarebbe un'utopia considerare che hanno la stessa efficacia, poiché il loro grado di efficienza è variabile. Quindi, non esiste una norma costituzionale destituita dall'efficacia. Tutte le disposizioni costituzionali hanno concretamente la possibilità di produrre, alla loro maniera, gli effetti giuridici da esse perseguiti." (DINIZ, Maria Helena. Norma constitucional e seus efeitos. 3. ed. São Paulo: Saraiva, 1997, p. 115).

${ }^{32}$ SILVA, José Afonso da. Aplicabilidade das normas constitucionais. 7. ed. Malheiros: São Paulo, 2008, p. 86. Nel diritto italiano risulta che la nozione di norma programmatica fu sviluppata da Vezio Crisafulli, a partire dal dibattito sull'efficacia delle norme costituzionali. (CRISAFULLI, Vezio. La costituzione e le sue disposizioni di principio. Milano: Giuffrè, 1952, apud BERCOVICI, Gilberto. Constituição econômica e desenvolvimento. Uma leitura a partir da Constituição de 1988. São Paulo: Malheiros, 2005, p. 39). Riccardo Guastini complementa dicendo che "una precisa linea di demarcazione tra norme di principio e norme programmatiche non vi è: le due categorie sfumano l'una nell'altra". (GUASTINI, Riccardo. Teoria e dogmatica delle fonti. Milano: Giuffrè, 1998, p. 355).

${ }^{33}$ MORAES, Guilherme Peña de. Direito constitucional. Teoria da Constituição. 3. ed. Rio de Janeiro: Lumen Juris, 2006, p. 83.
} 
dello Stato"), o schemi generali di strutturazione di istituzioni, organi o enti, come nel caso dell'articolo 91, comma 2 ("La legge regolerà l'organizzazione e il funzionamento del Consiglio di Difesa Nazionale"), lasciando al legislatore ordinario o ad altri organi di governo la sua realizzazione normativa che può, o meno, far riferimento a una legislazione futura. ${ }^{34}$

I diritti individuali sono enunciati secondo norme di applicabilità diretta e immediata, mentre le norme di definizione dei diritti sociali sono ancora, nella maggior parte, concepite come programmatiche. ${ }^{35}$ Considerato ciò, la dottrina cerca meccanismi costituzionali e fondamenti teorici per "superare il carattere astratto e incompleto delle norme di definizione dei diritti sociali". ${ }^{36}$ Esse configurano i diritti in modo incompleto, presentando bassa densità normativa.

In Italia, fin dall'entrata in vigore della Costituzione, la dottrina e la giurisprudenza si interrogano sulla definizione dei soggetti istituzionali che dovrebbero ritenersi giuridicamente obbligati a realizzare le condizioni di uguaglianza sostanziale, ovvero sulla definizione delle garanzie giurisdizionali che l'ordinamento predispone nei confronti dei diritti sociali di prestazione che i lavoratori e i soggetti economicamente o fisicamente più deboli vantano nei confronti dello Stato.

Sembra che il compito di determinare le forme, i modi e la misura, al di là del minimo essenziale, della concretizzazione di ogni simile pretesa soggettiva nei confronti dello Stato spetta, innanzitutto, al legislatore nazionale e regionale e, quindi, alla pubblica amministrazione dello Stato e delle regioni e agli enti locali. ${ }^{37}$

Secondo l'articolo 117, lettera "m" della Costituzione italiana, il dovere di determinare dei livelli essenziali delle prestazioni concernenti i diritti civili e sociali è del legislatore nazionale. La competenza statale è esclusiva, sebbene circoscritta alla determinazione dei livelli essenziali.

Questa previsione costituzionale costitui un vincolo per il legislatore statale e regionale, non solo sul piano del riparto di competenze normative "ma anche per ciò che

\footnotetext{
${ }^{34}$ SILVA, José Afonso da. Aplicabilidade das normas constitucionais. 7. ed. Malheiros: São Paulo, 2008, p. 84 e 123

${ }^{35}$ Viviana Molaschi, rifacendosi a R. Ferrara, dice che è innegabile il fatto che la nostra Costituzione si muova "eternamente sulla linea di confine fra norme programmatiche e norme immediatamente operative e quindi precettive", relativizzando molte delle sue statuizioni, ridotte a "spinte e stimoli di tipo orientativo". (MOLASCHI, Viviana. I rapporti di prestazione nei servizi sociali. Livelli essenziali delle prestazioni e situazioni giuridiche soggettive. Torino: Giappichelli, 2008, p. 58-59).

${ }^{36}$ SILVA, José Afonso da. Aplicabilidade das normas constitucionais. 7. ed. Malheiros: São Paulo, 2008, p. 140.

${ }^{37}$ GIORGIS, Andrea. Diritti sociali. In: CASSESE, Sabino (Coord.). Dizionario di Diritto Pubblico. V. III. Milano: Giuffrè, 2006, p. 1907.
} 
concerne il quid delle prestazioni, che devono concretizzare, quantomeno, il 'contenuto essenziale' dei diritti costituzionalmente tutelati". ${ }^{38}$

La competenza statale in ordine alla tutela delle prestazioni essenziali concernenti i diritti civili e sociali non opera soltanto a livello legislativo, bensì anche a livello amministrativo. ${ }^{39}$

La questione è se quelli che sono giuridicamente obbligati non adempiono al suddetto obbligo, o l'adempiono in maniera inadeguata. In questa situazione, ci si domanda se i giudici possono porre rimedio alle omissioni totali o parziali dei pubblici poteri. Le risposte della dottrina e della giurisprudenza a questa domanda sono avanzate nel corso del tempo.

Sappiamo che ai giudici non è dato attuare come "legislatori positivi"40, non rispettando il principio della tripartizione dei poteri. Però, in materia di minimo esistenziale, sosteniamo che la libertà di conformazione del legislatore è relativa. Quando la violazione a diritti minimi è evidente e arbitraria, crediamo che l'intervento giudiziale sarà autorizzato. ${ }^{41}$

L'articolo 3, comma 2 e gli altri articoli della Costituzione italiana che in maniera puntuale riconoscono agli individui più svantaggiati i diversi diritti sociali all'uguaglianza

\footnotetext{
${ }^{38}$ MOLASCHI, Viviana. I rapporti di prestazione nei servizi sociali. Livelli essenziali delle prestazioni e situazioni giuridiche soggettive. Torino: Giappichelli, 2008, p. 167-168. La Corte costituzionale ha precisato che "il legislatore può legittimamente imporre agli enti autonomi vincoli alle politiche di bilancio (ancorché si traducano, inevitabilmente, in limitazioni indirette all'autonomia di spesa degli enti), ma solo, con disciplina di principio, per ragioni di coordinamento finanziario connesse ad obiettivi nazionali, condizionati anche dagli obblighi comunitari". Questi vincoli saranno considerati rispettosi dell'autonomia delle regioni e degli enti locali solo in via transitoria ed in vista degli specifici obiettivi di riequilibrio della finanza pubblica perseguiti dal legislatore statale. In altri termini, la legge statale può stabilire solo un limite complessivo, che lasci agli enti stessi ampia libertà di allocazione delle risorse fra i diversi ambiti e obiettivi di spesa. (VERGOTTINI, Giuseppe de. Diritto Costituzionale. 6. ed. Padova: CEDAM, 2008, p. 739-740). In questo punto merita di essere trascritto il disposto dall'articolo 120, comma 2 della Costituzione italiana: "Il Governo può sostituirsi a organi delle Regioni, delle Città metropolitane, delle Province e dei Comuni nel caso di mancato rispetto di norme e trattati internazionali o della normativa comunitaria oppure di pericolo grave per l'incolumità e la sicurezza pubblica, ovvero quando lo richiedono la tutela dell'unità giuridica e dell'unità economica e in particolare la tutela dei livelli essenziali delle prestazioni concernenti i diritti civili e sociali, prescindendo dai confini territoriali dei governi locali. La legge definisce le procedure atte a garantire che i poteri sostitutivi siano esercitati nel rispetto del principio di sussidiarietà e del principio di leale collaborazione."

${ }^{39}$ Secondo LUCIANI, Fabrizio; ROLLI, Renato. Azione Amministrativa e Disciplina di Diritto Pubblico. Napoli: Edizioni Scientifiche Italiane, 2008, p. 460.

40 È necessaria una breve spiegazione sul concetto, utilizzato con frequenza in Brasile. Significa che ai giudici non è permesso creare norme primarie, in sostituzione del legislatore. Quindi, se nel realizzare il controllo di costituzionalità non è possibile estrarre da una legge o da un atto normativo un' "interpretazione secondo la Costituzione", ai giudici resterà solo la possibilità di dichiararne la sua nullità. (MORAES, Alexandre de. Direito Constitucional. 24. ed. São Paulo: Atlas, 2009, p. 16-17)

${ }^{41}$ La legittimità del controllo e l'intervento dei giudici in tema di politiche pubbliche richiede che sia presente "l'abusività governativa". Secondo Pedro Lenza, la violazione a diritti minimi deve essere evidente e arbitraria, come nel caso di spostamento del denaro destinato all'istruzione e alla sanità, in forza dell'articolo 34, comma VII, linea "e" della Costituzione brasiliana, per opere non necessarie come sono quelle di puro abbellimento. (LENZA, Pedro. Direito Constitucional Esquematizado. 14. ed. São Paulo: Saraiva, 2010, p. 253). In questa materia, è opportuno citare il principio della non esclusione del controllo giurisdizionale, consacrato nell'articolo $5^{\circ}$, comma XXXV della Costituzione federale: "la legge non escluderà dall'analisi dei giudici lesione o minaccia al diritto."
} 
sostanziale e ad una vita libera e dignitosa parrebbero infatti prescrivere ai giudici di ordinare alla pubblica amministrazione, che risulta preposta a garantire l'attuazione dello specifico principio costituzionale richiamato, di accollarsi il costo di detto bene o servizio, in maniera tale da assicurare, immediatamente, ai ricorrenti, l'effettivo esercizio del proprio fondamentale diritto. ${ }^{42}$

Infatti, la Corte costituzionale e i giudici comuni hanno ritenuto che la magistratura debba estrarre dalle disposizioni costituzionali scritte tutti i significati che in base alle regole della logica costituiscono loro possibili sviluppi e specificazioni.

La Corte d'Appello dell'Aquila, pochi anni dopo l'entrata in vigore della Carta costituzionale, nella pronuncia 24 ottobre 1950, proclamò che

costituirebbe un controsenso il poter supporre che una legge, anzi la legge fondamentale dello Stato, creata appositamente per determinare e per fissare i diritti e i doveri dei cittadini e, di conseguenza, additare e delimitare il campo e l'oggetto delle future leggi, non sia destinata ad una immediata e integrale attuazione, almeno fin dove ciò sia materialmente e giuricamente possibile. ${ }^{43}$

Quando si verifica che il legislatore non ha provveduto a definire neanche una minima articolazione amministrativa per il perseguimento dei diversi obiettivi sociali che il testo costituzionale prescrive siano realizzati dallo Stato, non sarebbe forse impossibile argomentare una responsabilità dello Stato. ${ }^{44}$

Sostenere diversamente sarebbe fare una interpretazione svalutativa della Costituzione, nel senso che le disposizioni costituzionali che riconoscono i diritti sociali a prestazioni positive non sono immediatamente applicabili, ovvero che l'intervento del legislatore sia giuridicamente indispensabile per definire il contenuto del diritto medesimo.

Pensare che la presenza di una puntuale disciplina legislativa sia una condicio sine qua non per l'effettiva azionabilità dei diritti sociali significherebbe, in ultima analisi,

42 GIORGIS, Andrea. Diritti sociali. In: CASSESE, Sabino (Coord.). Dizionario di Diritto Pubblico. V. III. Milano: Giuffrè, 2006, p. 1908.

${ }^{43}$ GIORGIS, Andrea. Diritti sociali. In: CASSESE, Sabino (Coord.). Dizionario di Diritto Pubblico. V. III. Milano: Giuffrè, 2006, p. 1908.

${ }^{44}$ GIORGIS, Andrea. Diritti sociali. In: CASSESE, Sabino (Coord.). Dizionario di Diritto Pubblico. V. III. Milano: Giuffrè, 2006, p. 1908-1909. Infine, "Anche se fondamentali e sanciti costituzionalmente, i diritti postulano sempre una legislazione d'attuazione, cioè il dover essere giuridico delle garanzie primarie rappresentate dai doveri corrispondenti, pena la loro indebita squalificazione come vuote declamazioni normative." (FERRAJOLI, Luigi. Principia iuris. Teoria del diritto e della democrazia. 1.Teoria del diritto. V. I. Bari: Laterza, 2007, p. 674). Lo stesso autore afferma che uno dei concetti centrali nella teoria dello stato costituzionale di diritto e uno dei vizi più gravi è quello di lacuna, che si verifica quando non osservato l'obbligo di produrre le norme giuridiche che soddisfano i diritti positivi costituzionalmente stabiliti oppure quando, "benché la costituzione preveda determinati diritti sociali, come il diritto alla salute o all'istruzione, non venga di fatto prodotta nessuna norma che disponga l'obbligo di soddisfarli e non siano istituiti gli apparati idonei alla loro soddisfazione nonché all'accertamento e alla riparazione delle loro violazioni'. (FERRAJOLI, Luigi. Principia iuris. Teoria del diritto e della democrazia. 1.Teoria del diritto. V. I. Bari: Laterza, 2007, p. 684 e 691). 
vanificare gran parte delle aspirazioni del diritto costituzionale contemporaneo. Affermare, in altri termini, l'esistenza di una riserva di legge anche in materia di definizione del contenuto costituzionalmente garantito del diritto di accedere ai beni e ai servizi essenziali significherebbe ribaltare la stessa ratio della riserva di legge: "quest'ultima, da strumento di garanzia dei diritti di libertà dei cittadini contro gli abusi dell'esecutivo, si tramuterebbe infatti in possibile strumento di abuso del legislatore contro i diritti dei più poveri" ${ }^{45}$

La dottrina, maggioritariamente, sostiene che

\begin{abstract}
Attualmente non è possibile negare il carattere giuridico e, quindi, l'esigibilità e l'azionabilità dei diritti fondamentali, nella sua molteplice tipologia. È puramente ideologica, e non scientifica, la resistenza che ancora oggi si oppone alla realizzazione, per via coercitiva, dei cosiddetti diritti sociali. Anche i diritti politici e individuali affrontarono, come segnalammo, la reazione conservatrice fino al loro consolidamento finale. L'affermazione dei diritti fondamentali nel loro insieme, nella loro piena applicazione, viene riconosciuta nelle Carte Politiche più recenti, come si vede nel'articolo 2 della Costituzione portoghese e dal Preambolo della Costituzione brasiliana, proclamando che il paese è uno Stato democratico, 'destinato ad assicurare l'esercizio dei diritti sociali e individuali' . ${ }^{46}$
\end{abstract}

Uno dei dibattiti più importanti nel Diritto Costituzionale odierno è quello relativo al vincolo dei Poteri Esecutivo, Legislativo e Giudiziario ai diritti fondamentali. L’importanza del tema è presentata dalle diverse questioni relative alla giustizia sociale del Brasile, specialmente la storica concentrazione del reddito. Non si può non decidere. Si considera che, anche quando non vi sarà applicabilità diretta in favore del titolare di un diritto fondamentale, ci sarà

il vincolo immediato rispetto al legislatore, che deve procedere alla regolamentazione del diritto, e anche nei confronti dei tribunali che devono obbligare il legislatore a implementare i diritti sociali e, eventualmente, sopperire alla mancanza mediante il controllo di costituzionalità delle omissioni legislative, il mandato di ingiunzione e alle altre garanzie fondamentali. ${ }^{47}$

Tuttavia, anche se l'azionabilità di diritti fondamentali sociali viene riconosciuta, non si può negare che la sua realizzazione è piu complessa di quella delle altre categorie. Sono innumerevoli le prestazioni esigibili dallo Stato, sia nella forma di consegna di un bene o nella soddisfazione di un interesse. La sua piena operatività trova ancora grande resistenza,

\footnotetext{
${ }^{45}$ GIORGIS, Andrea. Diritti sociali. In: CASSESE, Sabino (Coord.). Dizionario di Diritto Pubblico. V. III. Milano: Giuffrè, 2006, p. 1909.

${ }^{46}$ BARROSO, Luís Roberto. O direito constitucional e a efetividade de suas normas. 8. ed. Rio de Janeiro: Renovar, 2006, p. 102. Il dispositivo da egli citato, l'articolo 2 della Costituzione della Repubblica portoghese, proclama che essa è "uno Stato di diritto democratico basato sulla sovranità popolare; nel pluralismo di espressione e organizzazione politica democratiche, nel rispetto e nella garanzia del compimento dei diritti e libertà fondamentali e nella separazione e interdipendenza dei poteri, in vista della realizzazione della democrazia economica, sociale e culturale e l'approfondimento della democrazia partecipativa."

${ }^{47}$ DIMOULIS, Dimitri. Elementos e problemas da dogmática dos direitos fundamentais. In: SARLET, Ingo Wolfgang (coord). Jurisdição e direitos fundamentais. Porto Alegre: Livraria do Advogado, 2006, p. 84.
} 
nonostante il riconoscimento dell'indole normativa di tali disposizioni. Non bastassero i limiti economici alla completa implementazione di questi diritti, esistono inoltre quelli di carattere politico. Si consideri come esempio la questione dell'adozione della miglior misura possibile del carattere medico preventivo, tra le diverse esistenti, per contenere una determinata epidemia. Fatta eccezione per i casi estremi d'inerzia o di inadeguatezza evidente dei provvedimenti presi dallo Stato, tale decisione possiede un carattere eminentemente discrezionale, e quindi insindacabile in via giurisdizionale. ${ }^{48}$

Non a caso l'articolo 196 della Costituzione brasiliana non specifica quali politiche sociali ed economiche dovranno essere implementate per ridurre il rischio di malattie e per garantire l'accesso universale e ugualitario alle azioni e ai servizi per la diffusione, la protezione e il recupero della salute. La Costituzione italiana, allo stesso modo, non offre alcuna indicazione sull'organizzazione del servizio. ${ }^{49}$ Siccome la delimitazione del contenuto di questo dovere di protezione non è interamente stabilito nella Costituzione, risulta difficile il controllo dell'insufficienza, eccetto a ciò che riguarda le esigenze minime.

\section{La Costituzione brasiliana e il vincolo del bilancio pubblico con i diritti sociali}

La Costituzione della Repubblica Federativa del Brasile impone diversi limiti alla spesa pubblica. Così, nell'articolo 167, I, proibisce l'inizio di programmi o di progetti che non siano stati inseriti nella Legge di Bilancio Annuale.

La Legge Complementare Federale n. 101 del 04 maggio 2000, a sua volta, stabilisce norme di finanze pubbliche dirette alla responsabilità della gestione fiscale da applicarsi a tutti gli enti federativi. Secondo il testo del suo articolo 1,

\footnotetext{
Questa Legge Complementare stabilisce norme di finanze pubbliche dirette alla responsabilità nella gestione fiscale, in base al Capitolo II del Titolo VI della Costituzione.

$\S 1$ La responsabilità nella gestione fiscale presuppone l'azione pianificata e trasparente, nella quale si prevengono rischi e si correggono scostamenti capaci di intaccare l'equilibrio dei conti pubblici, mediante il raggiungimento di mete di risultati tra entrate e spese e il rispetto dei limiti e condizioni per quanto riguarda la rinuncia a entrate, creazione di spese col personale, della sicurezza sociale e altri, debiti consolidati e mobilia, operazioni di credito, compresi gli anticipi di entrate, concessione di garanzia e registrazione a Rimanenze a Pagare.

$\S 2$ Le disposizioni di questa Legge Complementare obbligano l'Unione, gli Stati, il Distretto Federale e i Comuni.
}

\footnotetext{
${ }^{48}$ BARROSO, Luís Roberto. O direito constitucional e a efetividade de suas normas. 8. ed. Rio de Janeiro: Renovar, 2006, p. 105.

${ }^{49}$ Neppure accenna ad una coesistenza o ad un concorso di pubblico e privato. (CORSO, Guido. Pubblico e privato nel sistema sanitario. In: FARES, Guerino (coord.). Il diritto alla salute tra istituzioni e società civile. Torino: Giappichelli, 2009, p. 17).
} 
Ancora sulla linea del controllo della spesa pubblica, si trova la proibizione, come regola generale, del vincolo dell'entrata di imposte a organo, a fondo o a spesa, come risulta all' articolo 167, IV, della Costituzione. ${ }^{50}$ Si tratta del principio di bilancio delle entrate non vincolate, il cui scopo è di evitare che sia ostacolato il finanziamento di spese generiche da parte del bilancio. Si osservi che la restrizione ricade, solamente, sulla modalità "imposta".

Tuttavia, il legislatore costituente stesso prevede eccezioni a questa regola. Per quanto riguarda questo dispositivo, nella sua parte conclusiva, con il testo risultante dall'emendamento costituzionale n. 42 del 19 dicembre 2003, fanno eccezione il prodotto delle entrate delle imposte secondo gli articoli 158 e 159, che si occupano, rispettivamente, della distribuzione obbligatoria agli Stati, al Distretto Federale e ai Comuni; le attività dell'amministrazione tributaria, come pure le risorse per la manutenzione e lo sviluppo dell'istruzione e per le azioni e servizi pubblici nell'area sanitaria.

Di conseguenza, trattandosi di istruzione o sanità, non esiste divieto al vincolo dell'entrata di imposte per tali fini, trattandosi di priorità dello Stato brasiliano.

Tant'è vero che l'articolo 198 della Costituzione determina:

$[\ldots]$

§ 2 L'Unione, gli Stati, il Distretto Federale e i Comuni applicheranno, annualmente, in azioni e servizi pubblici sanitari risorse minime derivate dall'applicazione di percentuali calcolate su: (Inserito dall'Emendamento Costituzionale n. 29 del 2000)

I - Nel caso dell'Unione, le entrate correnti nette del rispettivo esercizio finanziario non possono essere inferiori a 15\% (quindici per cento); (Testo redatto secondo l'Emendamento Costituzionale no 86 del 2015).

II - nel caso degli Stati e del Distretto Federale, il prodotto delle entrate delle imposte al quale si riferisce l'articolo 155 e delle risorse trattate agli articoli 157 e 159, I, lettera $a$, e II, dedotte le parti che saranno trasferite ai rispettivi Comuni; (Inserito nell'Emendamento Costituionale n. 29 del 2000)

III - nel caso dei Comuni e del Distretto Federale, il prodotto dell'esazione delle imposte al quale si riferisce l'articolo 156 e delle risorse trattate agli articoli 158 e 159 , I, lettera $b$ e $§ 3$. (Inserito dall'Emendamento Costituzionale n. 29 del 2000) $\S 3$ Legge Complementare, che sarà nuovamente analizzata perlomeno a ogni cinque anni, stabilirà: (Inserito dall'Emendamento Costituzionale n. 29 del 2000)

I - le percentuali trattate ai commi II e III del $\S 2^{\circ}$; (Testo redatto secondo l'Emendamento Costituzionale n $^{\circ} 86$ del 2015)

II - i criteri di divisione delle risorse dell'Unione vincolate alla sanità destinate agli Stati, al Distretto Federale e ai Comuni, e degli Stati destinate ai loro rispettivi Comuni, per una progressiva riduzione delle disparità regionali; (Inserito dall'Emendamento Costituzionale n. 29 del 2000)

${ }^{50} \mathrm{Fu}$ uno degli articoli della Costituzione che ha sofferto maggiori modifiche: prima con l'emendamento costituzionale n. 3/93, poi con quello n. 29/2000 e, in seguito, dal 42/2003. 
III - le norme di vigilanza, valutazione e controllo delle spese con la sanità nelle sfere federale, statale, distrettuale e comunale; (Inserito dall'Emendamento Costituzionale n. 29 del 2000)

Inspiegabilmente la legge complementare della quale tratta il comma 3 dell'articolo 198 non è stata ancora redatta.

Forse prevedendo il ritardo, lo stesso emendamento costituzionale, il n. 29 del 2000, ha aggiunto all'Atto delle Disposizioni Costituzionali Transitorie (ADCT), l'articolo 77 che, nel suo comma 4, stabilisce: "§ 4 In assenza della legge complementare alla quale si riferisce l'articolo 198, § 3, a partire dall'esercizio finanziario 2005, si applicherà all'Unione, agli Stati, al Distretto Federale e ai Comuni quanto disposto da questo articolo."

Così, le percentuali minime da applicare anualmente ad azioni e servizi pubblici sanitari sono ${ }^{51}$ :

Art. 77. Fino all'esercizio finanziario 2004, le risorse minime applicate alle azioni e servizi pubblici sanitari saranno equivalenti: (Inserito nell'Emendamento Costituzionale n. 29 del 2000)

I - nel caso dell'Unione: (Inserito nell'Emendamento Costituzionale n. 29 del 2000)

a) nell'anno 2000, il montante destinato ad azioni e servizi pubblici sanitari nell'esercizio finanziario 1999 aumentato, come minimo, del cinque per cento; (Inserito nell'Emendamento Costituzionale n. 29 del 2000)

b) dall'anno 2001 al 2004, il valore appurato nell'anno precedente, adeguato secondo la variazione nominale del Prodotto Interno Lordo - PIL; (Inserito nell'Emendamento Costituzionale n. 29 del 2000)

II - nel caso degli Stati e del Distretto Federale, dodici per cento del prodotto delle imposte raccolte al quale si riferisce l'art. 155 e delle risorse trattate dagli articoli 157 e 159, I, lettera $a$, e II, dedotte le parti trasferite ai rispettivi Comuni; e (Inserito nell'Emendamento Costituzionale n. 29 del 2000)

III - nel caso dei Comuni e del Distretto Federale, quindici per cento del prodotto delle imposte raccolte al qual si riferisce l'articolo 156 e delle risorse trattate dagli articoli 158 e 159, I, lettera $b$, e $\$ 3$. (Inserito nell'Emendamento Costituzionale n. 29 del 2000)

$\S 1$ Gli Stati, il Distretto Federale e i Comuni che applichino percentuali inferiori a quelle stabilite dai commi II e III dovranno aumentarli gradualmente, entro l'esercizio 2004, riducendo la differenza in ragione di, perlomeno, un quinto all'anno, considerando che, a partire dal 2000, l'applicazione sarà di perlomeno il sette per cento. (Inserito nell'Emendamento Costituzionale n. 29 del 2000)

$\S 2$ Delle risorse dell'Unione appurate secondo questo articolo, quindici per cento, come minimo, saranno applicate nei Comuni, secondo il criterio demografico, in azioni e servizi sanitari fondamentali, secondo la legge. (Inserito nell'Emendamento Costituzionale n. 29 del 2000)

$\S 3$ Le risorse degli Stati, del Distretto Federale e dei Comuni, destinate alle azioni e servizi pubblici sanitari, e quelle trasferite da parte dell'Unione per lo stesso scopo, saranno applicate per mezzo del Fondo alla Sanità, che sarà seguito e controllato dal Consiglio della Sanità, senza danneggiare quanto disposto dall'articolo 74 della Costituzione Federale. (Inserito nell'Emendamento Costituzionale n. 29 del 2000)

Nell'articolo 212 della Costituzione, si legge a proposito dell'istruzione:

\footnotetext{
51 Non dimenticando la modifica dell'articolo $198, \S 2^{\circ}$, comma I, della Costituzione federale, messa in
} atto dall'emendamento costituzionale $n=86 / 2015$. 
Art. 212. L'unione applicherà, annualmente, non meno del diciotto per cento, e gli Stati, il Distretto Federale e i Comuni venticinque per cento, come minimo, delle entrate risultanti dalle imposte, compresa quella proveniente da trasferimenti, nella manutenzione e sviluppo dell'istruzione.

$\S 1$ - La parte delle imposte raccolte trasferita dall'Unione agli Stati, al Distretto Federale e ai Comuni, o da parte degli Sati ai rispettivi Comuni, non è considerata, agli effetti di calcolo previsto in quest'articolo, come entrata del governo che la trasferisce.

$\S 2$ - Per effetto dell'applicazione di quanto disposto al "caput" di questo articolo, saranno considerati i sistemi di istruzione federale, statale e comunale e le risorse applicate secondo l'articolo 213.

§ 3 - La distribuzione delle risorse pubbliche assicurerà priorità all'assistenza delle necessità della scuola dell'obbligo, per quanto riguarda l'universalizzazione, garanzia di un livello di qualità ed equità, secondo i termini del piano nazionale per l'istruzione. (Testo risultante dall'Emendamento Costituzionale n. 59 del 2009)

$\S 4$ - I programmi supplementari di alimentazione e assistenza alla sanità previsti nell'articolo 208, VII, saranno finanziati con risorse provenienti da contributi sociali e altre risorse di bilancio.

$\S 5$ - L'istruzione pubblica fondamentale avrà come fonte aggiuntiva di finanziamento il contributo sociale dello "stipendio-istruzione", raccolto dale aziende, secondo la legge. (Testo risultante dall'Emendamento Costituzionale n. 53 del 2006)

Come si è visto, trattandosi di istruzione la stessa Costituzione stabilisce, rispetto agli enti federativi - Unione, Stati Membri, Distretto Federale e Comuni - le percentuali minime di risorse da investire. Mentre i Comuni attueranno dando priorità all'istruzione di base e a quella infantile, gli Stati e il Distretto Federale si occuperanno della scuola elementare e media, invece l'Unione "organizzerà il sistema federale di istruzione", secondo quanto disposto dall'articolo 211, caput e commi 1, 2 e 3 della Costituzione federale.

In materia di sanità, la Costituzione rimanda alla legge di natura complementare la stipula delle percentuali minime da applicare annualmente in azioni e servizi pubblici. L'esigenza di regolamentazione normativa è stata inserita nella Costituzione federale in forza di un emendamento, il n. 29, promulgato il 13 settembre 2000. È trascorso un tempo molto superiore al ragionevole, senza che la determinazione sia stata adottata. La lacuna non può essere colmata da un altro meccanismo integrativo. Si configura, quindi, un'omissione legislativa incostituzionale, rivestita di particolare gravità, trattandosi di sanită ${ }^{52}$. Solo se i diritti sociali vengono soddisfatti gli stessi diritti individuali sono di fatto garantiti. ${ }^{53}$ Tali

52 Nell'azione diretta di incostituzionalità per omissione $\mathrm{n}^{\mathrm{o}} 3.682$, proposta dall'Assemblea Legislativa dello Stato del Mato Grosso, dinanzi al Presidente della Repubblica e al Parlamento, il Supremo Tribunale Federale, presentando un appello al legislatore, ha stipulato un periodo (considerato ragionevole) di 18 mesi affinché l'articolo 18, paragrafo $4^{\circ}$ della Costituzione federale fosse regolamentato, attraverso una legge complementare. Quel Tribunale ha affermato che la inertia liberandi del Parlamento (della Camera e del Senato) può essere oggetto di azione diretta di incostituzionalità e che la mancata redazione della legge complementare durante un periodo ragionevole rappresenta una reale violazione dell'ordine costituzionale.

${ }^{53}$ FERRAJOLI, Luigi. Principia iuris. Teoria del diritto e della democrazia. V. 2. Bari: Laterza, 2007, p. 397. 
affermazioni ratificano il carattere di principio dei diritti fondamentali, come mandati di ottimizzazione.

La Costituzione brasiliana contiene una regola, già citata, determinando che le norme costituzionali hanno applicazione immediata. ${ }^{54}$ Malgrado suoni poco logico, la sua inclusione si giustifica dinanzi una pratica che, reiteratamente, nega tale evidenza. Anche la Germania e il Portogallo contemplano, nel loro testo costituzionale, una norma simile. ${ }^{55}$ In caso di mancato rispetto, la competenza per la loro applicazione spetta ai giudici. ${ }^{56}$

Vediamo ciò che avviene con il diritto all'istruzione. Nella Costituzione brasiliana, esso è previsto nelle seguenti norme:

Art. 205. L'istruzione, diritto di tutti e dovere dello Stato e della famiglia, sarà diffusa e incentivata con la collaborazione della società, mirando alla piena formazione della persona, alla sua preparazione all'esercizio della cittadinanza e alla sua qualifica al lavoro.

Art. 208. Il dovere dello Stato verso l'istruzione sarà effettuato mediante la garanzia di:

I - l'istruzione fondamentale obbligatoria e gratuita dai 4 (quattro) ai 17 (diciassette) anni d'età, assicurata anche la sua offerta gratuita a tutti coloro che a essa non hanno avuto accesso nell'età dovuta. (Testo dato dall'Emendamento Costituzionale n. 59 del 2009)

Lo stesso articolo 208 contiene, inoltre, due regole importanti: quella del comma 1, nel senso che l'accesso all'istruzione obbligatoria e gratuita è diritto pubblico soggettivo, e quella del comma 2, secondo la quale la mancata offerta d'istruzione obbligatoria da parte del Potere Pubblico, o la sua offerta irregolare, importa responsabilità dell'autorità competente, sanzione questa di natura politica.

\footnotetext{
${ }^{54}$ Secondo l'articolo 5, "Tutti sono uguali dinanzi alla legge, senza distinzione di qualsiasi natura, garantendo ai brasiliani e agli stranieri residenti nel Paese l'inviolabilità del diritto alla vita, alla libertà, all'uguaglianza, allla sicurezza e alla proprietà, secondo il testo a seguire: $\S 1$ - Le norme che definiscono i diritti e le garanzie fondamentali hanno applicazione immediata."

${ }^{55}$ L'articolo 1, comma 3, della Legge Fondamentale della Germania stabilisce che: "I diritti fondamentali qui enunciati costituiscono precetti giuridici direttamente applicabili, che vincolano i Poteri Legislativo, Esecutivo e Giudiziale." L'articolo 18, comma 1, della Costituzione portoghese stabilisce che "I precetti costituzionali relativi ai diritti, libertà e garanzie sono direttamente applicabili e vincolano gli enti pubblici e privati."

56 Secondo José Felipe Ledur, "prende corpo nella questione di bilancio il problema della sua mancata esecuzione da parte dell'Esecutivo, causando la riduzione quantitativa e qualitativa delle prestazioni sociali, il che, certamente, esige l'azione decisa del Pubblico Ministero, secondo imposizione della Legge n. 7.347/85. Di conseguenza, in questo caso i giudici non violeranno la loro competenza (aumento di spese non previste), ma eserciteranno un dovere costituzionale che li obbliga a imporre l'osservanza della legge (il bilancio è legge e, inoltre, quando considerata autorizzativa, lo sarà appena in parte) anche e soprattutto per i poteri della Repubblica."(LEDUR, José Felipe. Direitos fundamentais sociais. Efetivação no âmbito da democracia participativa. Porto Alegre: Livraria do Advogado, 2009, p. 99-100).
} 
Anche così, il diritto all'istruzione non ha avuto, in Brasile, piena concretizzazione. ${ }^{57}$

Se gli articoli menzionati assicurano il diritto, impongono il dovere e, inoltre, specificano l'obbligo e la gratuità dell'istruzione fondamentale, "solo per mancanza di serietà nei confronti della Costituzione si potrebbe dare a tali precetti l'interpretazione secondo la quale essi non attribuiscono all'individuo la possibilità di esigere una condotta da parte dello Stato". 58

Si impone l'azione decisa del Pubblico Ministero, istituzione che ha ricevuto dal legislatore costituente del 1988 rilevanti funzioni, non solo di accusa, ma anche di garanzia. ${ }^{59}$

Certamente è anche possibile

pensare sulla responsabilità personale dell' amministratore per il mancato rispetto dei parametri sopraccitati (perlomeno per malversazione, col rischio di ulteriori sanzioni).

Esistono altre richieste possibili, come, e.g., che si determini l'investimento obbligatorio delle risorse inizialmente destinate ad altre voci a politiche vincolate ai diritti fondamentali, se questo è ancora possibile dal punto di vista della fase di esecuzione del bilancio.

Sarebbe inoltre plausibile la richiesta di inserimento, nel bilancio dell'anno successivo, della differenza di risorse che avrebbe dovuto essere utilizzata nei diritti fondamentali e non lo è stata. ${ }^{60}$

Si ritiene che

Chi difende l'idea secondo la quale non spetta ai giudici interferire nella questione che, per le sue caratteristiche, è essenzialmente politica ed economica, deve difendere, di conseguenza, la soppressione del dispositivo o la sua trasformazione in regola programmatica. Quello che sfida la serietà con cui deve essere trattata la Costituzione è il raziocinio secondo cui non vale ciò che è scritto. ${ }^{61}$

\footnotetext{
57 In Italia, invece, la Costituzione, nei suoi articoli 33 e 34, stabilisce: “[...] La Repubblica detta le norme generali sull'istruzione ed istituisce scuole statali per tutti gli ordini e gradi”; "34. La scuola è aperta a tutti. L'istruzione inferiore, impartita per almeno otto anni, è obbligatoria e gratuita. I capaci e meritevoli, anche se privi di mezzi, hanno diritto di raggiungere i gradi più alti degli studi. La Repubblica rende effettivo questo diritto con borse di studio, assegni alle famiglie ed altre provvidenze, che devono essere attribuite per concorso." Quanto a questi dispositivi, si deve chiarire che, per quanto la Costituzione prescriva solo otto anni di istruzione obbligatoria, la legislazione ordinaria, il decreto legge 15 aprile 2005, n. 76, prevede che la Repubblica assicura a tutti il diritto all'istruzione e alla formazione per almeno dodici anni o, comunque, sino al conseguimento di una qualifica di durata almeno triennale entro il diciottesimo anno di età. (Leggere la Costituzione Repubblicana. Breve commento articolo per articolo. IV ed. Napoli: Simone, 2007, p. 41).

${ }^{58}$ BARROSO, Luís Roberto. O direito constitucional e a efetividade de suas normas. Limites e possibilidades da Constituição Brasileira. 8. ed. Rio de Janeiro: Renovar, 2006, p. 143-144.

${ }^{59}$ Nell'articolo 129, III, della Costituzione federale si legge: “Art. 129. Sono funzioni istituzionali del Pubblico Ministero: III - promuovere l'indagine civile e l'azione civile pubblica, per la protezione del patrimonio pubblico e sociale, dell'ambiente e di altri interessi diffusi e collettivi."

${ }^{60}$ BARCELLOS, Ana Paula. Constitucionalização das políticas públicas em matéria de direitos fundamentais: o controle político-social e o controle jurídico no espaço democrático. In: SARLET, Ingo Wolfgang; TIMM, Luciano Benetti (org.), Direitos fundamentais e reserva do possível. Porto Alegre: Livraria do Advogado, 2008, p. 132-133.

${ }^{61}$ BARROSO, Luís Roberto. O direito constitucional e a efetividade de suas normas. Limites e possibilidades da Constituição Brasileira. 8. ed. Rio de Janeiro: Renovar, 2006, p. 150.
} 
Inoltre, la funzione giurisdizionale non può essere appena restauratrice, ma di formulazione e di attuazione pratica delle norme concrete che devono disciplinare una determinata situazione. ${ }^{62}$

\section{Considerazioni finali}

Il tema scelto è fonte di inquietudine intellettuale in tutto il mondo. Esiste una profusione di lavori teorici sull'efficacia dei diritti fondamentali sociali e sul ruolo dei pubblici poteri nello Stato Costituzionale. La tensione che si installò tra il diritto e la politica ha fomentato il dibattito accademico.

Nella parte iniziale del lavoro è stato messo in evidenza che lo Stato possiede un carattere strumentale, il cui fine ultimo è quello di propiziare che tutti gli individui raggiungano il loro completo sviluppo.

Si ritiene che la massimizzazione del benessere può essere ottenuta soltanto attraverso l'effettiva partecipazione dello Stato, sotto forma di prestazione di servizi pubblici di qualità.

La sua azione sostanziale, tendente ad assicurare a ciascun individuo perlomeno un minimo in prestazioni, caratterizza lo Stato Sociale e Democratico di Diritto, che rappresenta l'apice di una lenta evoluzione.

In questo modello, si attribuisce allo Stato un comportamento attivo nella realizzazione della giustizia sociale.

Così come lo Stato, anche i diritti fondamentali subirono trasformazioni. Il loro sviluppo non seguì un percorso lineare, cambiando secondo le esperienze statali.

La storia del Brasile, in particolare, fu marcata dalla dominazione coloniale; dal regime totalitario denominato di "Stato Nuovo", che si estese dal 1937 al 1945; dal colpo di stato militare del 1964, i cui effetti continuarono fino al 1985; dall'impeachment del primo presidente eletto democraticamente dopo questo periodo.

Come se non bastasse, ebbe sei Costituzioni, tra cui quella del 1988, che malgrado estremamente avanzata, ha già sofferto più di ottanta emendamenti.

\footnotetext{
${ }^{62}$ Secondo José Carlos Barbosa Moreira, O novo processo civil brasileiro, 1980, p. 11, apud BARROSO, Luís Roberto. $O$ direito constitucional e a efetividade de suas normas. Limites e possibilidades da Constituição Brasileira. 8. ed. Rio de Janeiro: Renovar, 2006, p. 121.
} 
Visti questi fatti, non sorprende che il paese presenti una fisionomia immatura, frutto delle successive rotture istituzionali. Le manca una società civile organizzata e un sentimento costituzionale.

Malgrado figuri nel panorama mondiale come uno dei grandi produttori di ricchezza, il Brasile continua a presentare indici insoddisfacenti in aree essenziali come l'istruzione, la sanità e l'abitazione. Le intense disuguaglianze socioeconomiche hanno come conseguenza la miseria, la violenza, l'inefficienza dell'apparato statale, l'apatia politica, insomma, una democrazia di ridotta qualità.

Sorprende che l'essenzialità dei diritti sociali sia stata per molto tempo contestata sia in Italia che in Brasile e anche in altri ordinamenti giuridici. Gli autori che anche oggi si oppongono al loro riconoscimento come autentici diritti fondamentali sostengono che la maggior parte dei diritti sociali è enunciata per mezzo di norme programmatiche e che per questo sono sprovvisti di vincolo giuridico, rappresentando un mero appello alle istanze politiche. Ci sono anche quelli che riducono il loro carico di essenzialità appena al cosiddetto minimo esistenziale.

È possibile affermare che tale discussione è superata. Oggi si sa che i diritti sociali rientrano a pieno titolo nel novero dei diritti fondamentali e che costituiscono anche elementi qualificanti dello Stato sociale democratico di diritto. Da ciò la duplice natura dei diritti sociali, i quali rappresentano, nel medesimo tempo, diritti veri e propri e disposizioni costituzionali di principio.

La giurisprudenza e la dottrina italiana e brasiliana prevalenti riconoscono ai diritti sociali il carattere di diritti inviolabili o fondamentali.

L’oggetto tipico dei diritti sociali è il cosiddetto "minimo di esistenza materiale", come misura di minor grado di protezione nelle aree di educazione e sanità, per esempio. Tuttavia, lo Stato deve diffondere l'incremento delle condizioni di vita oltre questo livello.

I soggetti istituzionali giuridicamente obbligati a realizzare le condizioni di uguaglianza sostanziale sono il legislatore e la Pubblica Amministrazione. La questione è se essi non adempiono al suddetto obbligo, o l'adempiono in maniera inadeguata. In questa situazione, ci si domanda se i giudici possono porre rimedio alle omissioni totali o parziali dei pubblici poteri. Sappiamo che ai giudici non è dato attuare come "legislatori positivi", non rispettando il principio della tripartizione dei poteri. Però, quando la violazione a diritti minimi è evidente e arbitraria, crediamo che l'intervento giudiziale sarà autorizzato. Le garanzie dei diritti si manifestano soprattutto attraverso l'operare della giurisdizione. 
Sostenere il contrario sarebbe fare una interpretazione riduttiva della Costituzione, nel senso che le disposizioni costituzionali che riconoscono i diritti sociali a prestazioni positive non sono immediatamente applicabili, ovvero che l'intervento del legislatore sia giuridicamente indispensabile per definire il contenuto del diritto medesimo. È puramente ideologica, e non scientifica, la resistenza che ancora oggi si oppone alla realizzazione, per via coercitiva, dei cosiddetti diritti sociali.

Con l'intuito di conferire efficacia ai diritti sociali, la Costituzione brasiliana fissa investimenti minimi obbligatori di risorse di bilancio per la sanità e l'istruzione, come si deduce dagli articoli 198, commi 2 e 3, e 212, commi dall'1 al 6.

Pertanto, la discrezionalità del legislatore non è assoluta. I Poteri Legislativo e Esecutivo devono, nel decidere sull'uso delle entrate, dare priorità ai diritti sociali, soprattutto quelli dell'istruzione e della sanità. 